\title{
EMA beamline status and its XRD prospects
}

\author{
G. A. Calligaris, M. A. S. Eleotério, J. C. Corsaletti Filho, H. H. V. L. Campos, \\ J. A. V. Mendonça, C. Doro, A. D. Grockowiak, U. F. Kaneko, R. D. dos Reis, N. M. Souza-Neto
}

Brazilian Synchrotron Light Laboratory, Brazilian Center for Research in Energy and Materials, Campinas, SP, 13083-970, Brazil

guilherme.calligaris@lnls.br

The EMA (Extreme condition Methods of Analysis) is one of the first Sirius beamlines, the 4th generation Brazilian synchrotron source. Currently under commissioning, its focus is on merging extreme thermodynamic conditions with a solid characterization platform based on spectroscopy and scattering techniques. For this, it has an undulator source and optics based on a high-dynamic double-crystal monochromator (HD-DCM) [1], 1/4 wave plates (double phase retarder), and KB-mirrors, which can provide X-ray beam sizes as small as $\sim 1 \times 0.5 \mu \mathrm{m}^{2}$ and $\sim 100 \mathrm{~nm}^{2}$, respectively, for two experimental stations (Microfocus and Nanofocus hutches).

Here is shown the current developments for the XRD experiments performed on both the "multipurpose setup" (@ $45 \mathrm{~m}$ from the source) and the 6-circle diffractometer (@55 m) [2]. The former relies on a hexapod for sample positioning and angular scans and offers the most extreme temperature $(0.5-5000 \mathrm{~K})$ and pressure $(\sim 600 \mathrm{GPa})$ within the Microfocus hutch, allied with a $1 \mathrm{~T}$ magnet. The latter setup will work with tunable beam sizes ranging from $\sim 13 \times 3$ up to $\sim 300 \times 300 \mu \mathrm{m}^{2}$ and positioning systems on top of its inner circle that delivers up to $66 \mathrm{~mm}$ of free working distance, expanding the possibilities for third-party sample mountings and environments, such as an available uniaxial strain cell. For this setup, the significant range in temperature $(5-300 \mathrm{~K})$, pressure $(\sim 300$ $\mathrm{GPa}$ ), and magnetic field (6T) add flexibility to the already versatile 4S+2D diffractometer. Additionally, opportunities for a broad sort of techniques supported at EMA will be discussed for powder and single-crystalline samples.

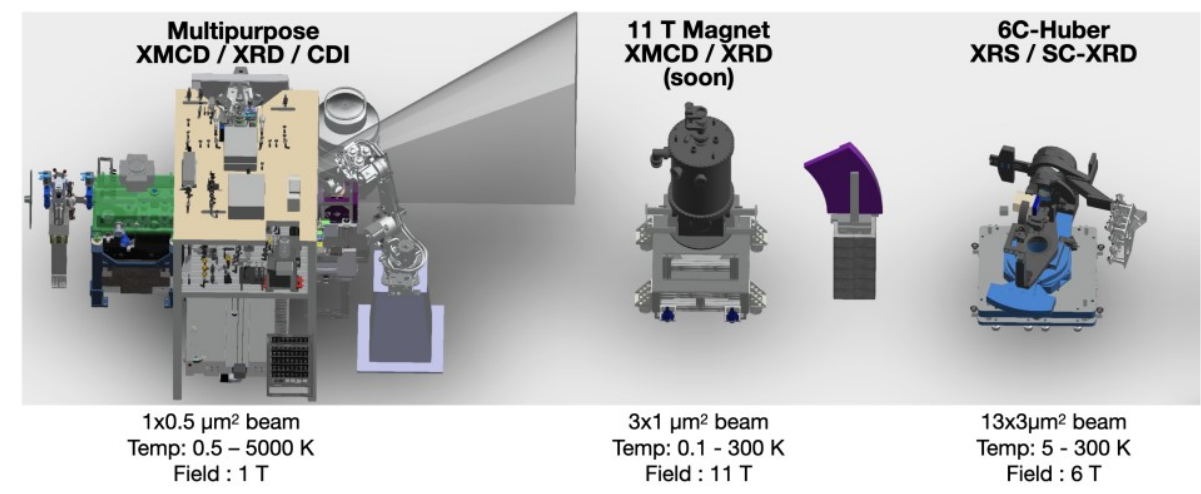

Figure 1. The three Microfocus hutch experimental setups, of which the Multipurpose and 6C-diffractometer are discussed here.

[1] Geraldes, R. R., et al. The Status of the New High-Dynamic Dcm for Sirius. 2018. Geneva, Switzerland: JACoW Publishing.

[2] dos Reis, R. D., et al., Journal of Physics: Conference Series, (2020), 1609, 012015.

Keywords: XRD; Extreme conditions beamline; 4th generation synchrotron 